\title{
A006
}

\section{Business-focused Play Analysis}

\section{D.G. Quirk* (Maersk Oil), D.J. Kann (Maersk Oil), R. Clark (Maersk Oil), E. Willerslev (Maersk Oil), M. Howe (Maersk Oil), M. Nielsen (Maersk Oil) \& O. Nykjaer (Maersk Oil)}

\section{SUMMARY}

Over the last few years, Maersk has developed a play analysis technique aimed directly at helping the company make decisions on where to explore by identifying areas of high prospectivity, predicting future discoveries within these areas, estimating finding costs and economic value and comparing to other regions in a consistent way.

The technique involves screening regional data and historical drilling results, delineating sweetspots, quantifying the undrilled potential and high grading those areas that rank highly based on a set of criteria, including commercial risk, size of prize, yet-to-find (YTF) richness and profit (Figure 1). Our experience is that better technical evaluations come out of play analyses where the emphasis is on understanding hydrocarbon charge, gross structure, thickness trends, number and size of traps and economics than those focusing on facies and depositional environments.

The process has proved to be efficient and allows technical staff to quickly focus on the key issues in high graded areas, particularly on identifying and evaluating new prospects and comparing them to features already drilled. The ranking and attractiveness of individual sweetspots is continually reassessed on this basis. 
Over the last few years, Maersk has developed a play analysis technique aimed directly at helping the company make decisions on where to explore by identifying areas of high prospectivity, predicting future discoveries within these areas, estimating finding costs and economic value and comparing to other regions in a consistent way.

The technique involves screening regional data and historical drilling results, delineating sweetspots, quantifying the undrilled potential and high grading those areas that rank highly based on a set of criteria, including commercial risk, size of prize, yet-to-find (YTF) richness and profit (Figure 1). Our experience is that better technical evaluations come out of play analyses where the emphasis is on understanding hydrocarbon charge, gross structure, thickness trends, number and size of traps and economics than those focusing on facies and depositional environments.

The process has proved to be efficient and allows technical staff to quickly focus on the key issues in high graded areas, particularly on identifying and evaluating new prospects and comparing them to features already drilled. The ranking and attractiveness of individual sweetspots is continually reassessed on this basis.

The presentation will show a typical workflow using ArcGIS and Excel on key maps and information, prioritized to reflect the time and data available, to get a final range in YTF resource and potential value.

Figure 1. The bare bones of focused play analysis in Maersk

A pragmatic, minimum requirement for play analysis in MOG involves the following:

1. Define \& name play (e.g. country, basin, age, res type using naming convention) - not necessary to split into multiple stratigraphic intervals

- define main trap type

- use ArcGIS

2. Decide area of interest \& outline sweetspot (where do we want to be): - generally lowest risk area (kitchen) \&/or possible extension containing leads - defined on key geological trends (burial depth, thickness, structural elements)

3. Estimate future chance of success (e.g. same as historical) separated into: - part play risk (proven vs. unproven, chance of all elements working in $\geq 1$ place) - average prospect risk (conditional on part play working)

4. Estimate number of leads/prospects in part play (things we want to drill) \& predict no. of discoveries based on average prospect risk, assuming part play works

5. Predict future discovery size distribution using historical or analogue P99 (lowside) \& mean size of good prospect (upside), assuming lognormality

6. Calculate range in yet-to-find (YTF) recoverable resource

7. Use MEFS \& NPV/bbl to predict economic YTF \& value

8. Normalise YTF \& economic value to standard area for comparison purposes 